\title{
On the 10th Anniversary of ACM's Digital Library
}

When ACM launched its pioneering Digital Library

a decade ago, it was one of the first professional societies to offer its members - and the broader computing community - a digital repository of its publications.

At launch, the ACM Digital Library contained the full text of all articles published by ACM from 1991 forward, and the metadata for articles published back to 1985 .

ACM's Digital Library proved a success from the outset. The decision to make the metadata for ACM's digital collection freely available (with subscriptions only required for downloading full text) allowed the computing community worldwide to use and benefit from the Digital Library regardless of their relationship with ACM. That decision, combined with extremely affordable pricing for individuals and institutions to access the full text of ACM articles, set the stage for early, enthusiastic engagement of the Digital Library.

Since its launch, ACM has maintained an ongoing commitment in time, talent, and investment to en-

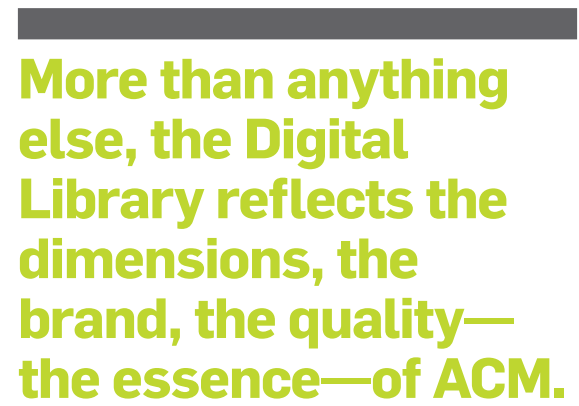

sure the Digital Library continues to flourish and fulfill the needs of the community. Within a year of its debut (and earlier than other professional societies), ACM resolved to capture and host everything the organization had ever published. We also decided to raise the visibility and importance of The Guide to Computing Literature, ACM's bibliographic database of the computing citations from a vast array of global publishers. And we elected to extract references from all publications (electronic and scanned) and treat them as first-class metadata. These decisions were at the core of a major reimplementation of the Digital Library released in 2001. That release included reference linking across all ACM publications, citation counts for ACM articles, and a significantly enhanced Guide.

The Digital Library, and the role it plays within the computing community, are top priorities for ACM. Throughout the last decade we have made significant investments in its content, features, performance, and worldwide reach. As a result, the Digital Library is now available at over 2,500 institutions around the globe; 26,000 professional members and 14,000 student members hold individual subscriptions. There are over 2.5 million unique visits per month, one million articles downloaded each month, and 75,000 Digital Library searches conducted each day.

ACM will continue investing in the
Digital Library. New search technology recently integrated has dramatically enhanced Digital Library searches and will enable a much richer, guided navigation experience of ACM (and other publishers') content. A major effort has been completed to normalize author and institution names so the community can easily and accurately find the published work of specific authors and institutions. New bibliometrics are now associated with each article and aggregated for authors (and soon institutions). The new ACM Author Page shows the collected works of an author, institutional affiliations, as well as individual and aggregate citation and download counts. With these new features, users can easily see not only who is publishing, but which articles are actually being downloaded (and presumably read).

The Digital Library has become ACM's most significant product and service. More than anything else, the Digital Library reflects the dimensions, the brand, the quality - the essenceof ACM. The success of the Digital Library, however, doesn't stop here. ACM will continue to invest resources and talent to ensure its Digital Library stays at the leading edge, is accessible and affordable to everyone, and remains the premier digital repository for the computing community.

\section{John R. White}

CHIEF EXECUTIVE OFFICER, ACM 